\title{
FOCO Y TÓPICO EN LOS ENUNCIADOS QUECHUAS: UN REPASO A LA GRAMÁTICA FUNCIONAL DEL RUNASIMI
}

\section{Focus and topic in Quechua sentences: a review of functional grammar of runasimi}

\author{
Niel A. Palomino Gonzales $1, \mathrm{a}$, : \\ ${ }^{1}$ Universidad Andina del Cusco, Cusco, Perú \\ ${ }^{\mathrm{a}}$ Magister \\ $\equiv$ npalomino@uandina.edu.pe
}

\begin{abstract}
Resumen
En la lingüística quechua, poco se ha explorado el tema de la 'gramática funcional'. En tal sentido, el presente artículo tiene por objetivo realizar un repaso sobre la gramática funcional del quechua; es decir, explora, muestra y explica las funciones comunicativas y pragmáticas de tópico/comento, soporte/aporte y el fenómeno de focalización en los enunciados quechuas, se consideraron treinta y dos enunciados en lengua quechua. El método empleado es el deductivo-hipotético; pues se parte de la teoría conocida y se termina en el ejemplo específico: enunciados quechuas. Como resultado se tiene que, en el quechua, el tópico es marcado por los sufijos - qa y -ri, y los focalizadores o marcadores de relieve son los sufijos -pas, -mi, -lla (n), - ña, -ta (n), -paq, - pi (n), -wan; entre otros. Y la conclusión primordial es que, desde el punto de vista de la gramática funcional, el quechua es una lengua en la que es posible encontrar unidades lingüísticas con función de soporte/aporte y tópico/comento, de manera muy frecuente y notoria. Asimismo, el quechua se puede tipificar como una lengua con tópico no gramaticalizado y con sujeto conocido, al igual que en el español. Estos resultados se pueden aplicar en el aprendizaje - enseñanza del idioma quechua para que los estudiantes puedan focalizar y localizar sus enunciados con propiedad lingüística.
\end{abstract}

Palabras clave: Foco, tópico, tema, rema, soporte, aporte, sintaxis quechua

\begin{abstract}
In the Quechua linguistics little has been explored the topic of the functional grammar. To this respect, this article tries to realize a revision to the functional grammar of Quechua; that is, it explores, shows and explains the communicative and pragmatic functions of topic / comment, support / contribution and the phenomenon of focusing on Quechua sentences. As participants, have been considered thirty-two statements in the Quechua language. The used method is deductively - hypothetic; So, it begins with the general theory and finishes in the specific example: Quechua statement. As a result we have that, in Quechua, the topic is marked by the suffixes -qa and -ri and the focalizers or focus markers are the suffixes -pas, -mi, -lla (n), -ña, -ta (n ), -paq, -pi (n), -wan, among others. And the main conclusion is that, from the point of view of functional grammar, Quechua is a language in which it is possible to find linguistic units with support/contribucion function and topic/comment in a very frequent and notorious way. Likewise, Quechua can be typified as a language with a non-grammatical topic and a known subject, as well as Spanish. These results can be applied to learning - teaching the Quechua language so that students can focus and topicalizer their utterances with linguistic property.

Keywords: Focus, topic, topic, row, support, input, Quechua syntax
\end{abstract}

Citar como: Palomino, N. (2018). Foco y tópico en los enunciados quechuas: un repaso a la gramática funcional del runasimi. Rev Yachay, 7(1), 381-388.

Recibido: 16-09-2018; Aceptado 07-12-2018

\section{Introducción}

En 1959, el lingüista francés Lucien Tesniere en su libro póstumo Elementos de sintaxis estructural, propuso un modelo de análisis gramatical denominado 'gramática valencial', o 'gramática de las dependencias'. Dos aspectos resaltan en este modelo: primero, un cuestionamiento al análisis estructuralista centrado únicamente en las palabras aisladas de la oración. Segundo, el análisis de las relaciones o conexiones de dependencias entre las palabras que integran un enunciado. Para Tesniere, el vínculo de las palabras en una oración se da de manera jerárquica en el cual, el subordinador es llamado regente y el subordinado es el regido. Los elementos regidos constituyen —en esa relación de dependencias - un nudo central. Los elementos del nudo pueden ser imprescindibles, llamados actuantes de la acción y otros son elementos prescindibles denominados circunstantes.

Por su parte, en la década de los 60 del siglo pasado, el lingüista holandés Simon C. Dik, propuso otro modelo de análisis oracional, similar al modelo de Tesniere. A este modelo se le llamó 'lingüística funcional'. Según Dik, la lengua es un instrumento de interacción social; es 
decir, tiene como función suprema la comunicación. Entonces, según esta gramática hay que analizar o estudiar a la lengua en su uso, es decir, en su función comunicativa. Estos modelos de análisis - la de Tesniere y Dik-, cimentaron las bases de la «Gramática generativa transformacional» de Chomsky, y abrieron camino para la pragmática y la lingüística textual.

Pero, estos avances en el campo de la gramática no han sido debidamente aprovechados por los lingüistas quechuas o estudiosos del quechua. Ahí el problema central de la lingüística quechua, cuyo estudio persiste en la más antigua y obsoleta gramática. Por todo ello, el objetivo primordial de este artículo es realizar una revisión exhaustiva a la gramática quechua según los cánones de la gramática funcional. La hipótesis que defendemos es: que, en los enunciados quechuas, como en todas las lenguas, se cumplen las funciones comunicativas y pragmáticas de tópico/comento, soporte/aporte y el fenómeno de focalización. El presente trabajo contribuye a un mayor conocimiento sobre la gramática quechua para así facilitar su aprendizaje pues, cuando se conoce la estructura y funcionamiento de una lengua es más fácil su aprendizaje.

Sobre este tema de focalización y topicalización, o gramática funcional, del quechua no hemos hallado antecedentes, solo estudios aislados de Cerrón - Palomino, de Antonio Cusihuamán y Julio Calvo. Por tal motivo, con esta investigación pretendemos contribuir a un mayor y mejor conocimiento de la estructura o gramática del quechua.

Efectivamente, Simon Dik, en su libro Gramática funcional (1981; 136 - 137), reconoce y propone dos funciones pragmáticas para especificar el estatus informativo de los constituyentes de la oración con relación al marco comunicativo en que son utilizados. Estas funciones pragmáticas son:

- Funciones intraoracionales: tópico y foco. (De carácter paradigmático)

- Funciones extraoracionales: tema o soporte y rema o aporte. (De carácter sintagmático).

Simon Dik y los integrantes de la 'escuela funcionalista' de Ámsterdam: J. T. Platt, W. A. Cook; se dieron cuenta de que las oraciones o enunciados informativos son respuestas a una serie de preguntas tácitas. En este sentido, las oraciones enunciativas constituyen la información conocida; por su parte la pregunta $-\mathrm{y}$ la respuesta a dicha pregunta-, constituye la información desconocida o nueva. Ejemplo:

\section{PREGUNTA}

\section{RESPUESTA}

\begin{tabular}{|c|c|c|c|}
\hline APORTE & SOPORTE & SOPORTE & APORTE \\
\hline ¿Quién & Escribió la canción Valicha? & La canción Valicha la escribió & Miguel Ángel Hurtado \\
\hline
\end{tabular}

Al respecto, para Mendenhall, citado por Salvador Gutiérrez (1997), en la estructura informativa se pueden distinguir dos funciones: soporte/aporte.

a) La función soporte. Llamada también tema, es todo aquello que nos transmite la información conocida supuesta, o supuestamente conocida. Responde a los interrogativos.

b) La función aporte. Denominada también rema, es todo aquello que contiene la información novedosa, supuestamente desconocida por el interlocutor.

La identificación del soporte o aporte se precisa a través de una serie de preguntas y respuestas. Ejemplos:

\begin{tabular}{llll}
\hline \multicolumn{1}{c}{ Pregunta } & \multicolumn{2}{c}{ Respuesta } \\
\hline ¿Porte & \multicolumn{1}{c}{ Soporte } & \multicolumn{1}{c}{ Soporte } & Aporte \\
\hline ¿Quién & Valicha takita qilqaran? & Valicha takitaqa qilqaran & Miguel Ángel Hurtado \\
& Escribió la canción Valicha? & La canción Valicha escribió & Miguel Ángel Hurtado \\
¿Imatan & Miguel Ángel Hurtado qilqaran? & Miguel Ángel Hurtado qilqaran & Valicha takita \\
¿Qué & Escribió Miguel Angel Hurtado? & Miguel Angel Hurtado escribió & la canción Valicha \\
¿Maymantataq & Karan Miguel Angel Hurtadori? & Miguel Angel Hurtado karan & Acopiyamantan \\
¿De dónde & Era Miguel Ángel Hurtado? & Miguel Ángel Hurtado era & de Acopía \\
\hline
\end{tabular}


Y la diferencia consustancial entre soporte y aporte radica en que la información nueva o aporte, y solo ella, puede aceptar una negación. No A, sino B; mientras que el soporte no acepta dicha negación. Ejemplo:

- Manan Alomía Robleschu, Miguel Ángel Hurtadun Valicha takitaqa qilqaran. (Miguel Ángel Hurtadun, es aporte). No Alomía Robles, sino, Miguel Ángel Hurtado escribió la canción Valicha.

- Mana Kuntur pasatachu, Valicha takipunin Miguel Ángel Hurtaduqa qilqasqa. (Valicha takipunin, es aporte). No, El cóndor pasa, sino Valicha fue escrita por Miguel Ángel Hurtado.

- Manan Sanqararamantachu, Aqupiyamantan Miguel Ángel Hurtaduqa karan. (Aqupiyamantan es aporte). No de Sangarará, sino de Acopía era Valicha.

\section{Materiales y métodos}

Los materiales empleados en esta investigación fueron los textos escritos quechuas como Gregorio Condori y Asunta Mamani, de Valderrama y Escalante; otros textos grabados de los quechuahablantes y otros creados por el mismos autor.

La metodología empleada es el deductivo-hipotético. Los conceptos de los temas centrales los exponemos en castellano y luego completamos los ejemplos con los enunciados quechuas.

\section{Resultados}

\section{El tópico, marco o circunstante}

Concepto. En lingüística, se denomina 'tópico' a una palabra o frase que presenta a una oración y señala algún contexto o circunstancia en el cual se realiza la oración o idea que se expresa. Como se verá más adelante, el tópico está separado de la misma oración y su presencia en la oración no es necesaria. Los lingüistas alemanes han llamado al tópico como 'sujeto psicológico'. Según ellos, el sujeto psicológico, es aquello en el cual el declarante de la oración quiere hacer pensar al oyente, antes de decirle la oración. Así, por ejemplo, si digo: «En lo referente a Kilku Warak'a, él escribió hermosos poemas en quechua». Los componentes de esta expresión se organizarían de la siguiente forma:

Tabla 1:

Componentes de una oración según la gramática funcional

\begin{tabular}{ccc}
\hline \multicolumn{1}{c}{ Tópico } & \multicolumn{2}{c}{ Comento } \\
\cline { 2 - 3 } (Sujeto psicológico) & $\begin{array}{c}\text { Tema } \\
\text { (Sujeto real) }\end{array}$ \\
\hline En lo referente a Kilku Warak'a & él & escribió hermosos poemas en quechua
\end{tabular}

De la misma forma, sobre el tópico, Luna Traill, E.; Vigueras Á. A.; Báez P. G. (2005) dicen:

Para algunos lingüistas el tópico y el comentario son correspondientes a la dicotomía tradicional de sujeto y predicado. Otros han especificado también que lo que funciona como tópico no es el sujeto gramatical sino el psicológico. Es cierto que los tópicos suelen constituirse por temas, pero no necesariamente coinciden con temas porque no todo tema constituye tópico.

Por otra parte, como se puede notar en los párrafos anteriores, el tópico viene dicotómicamente vinculado con el comento. Este último, comento o comentario, es toda la oración completa constituida por un tema y un rema, tal como presentamos en este esquema. 


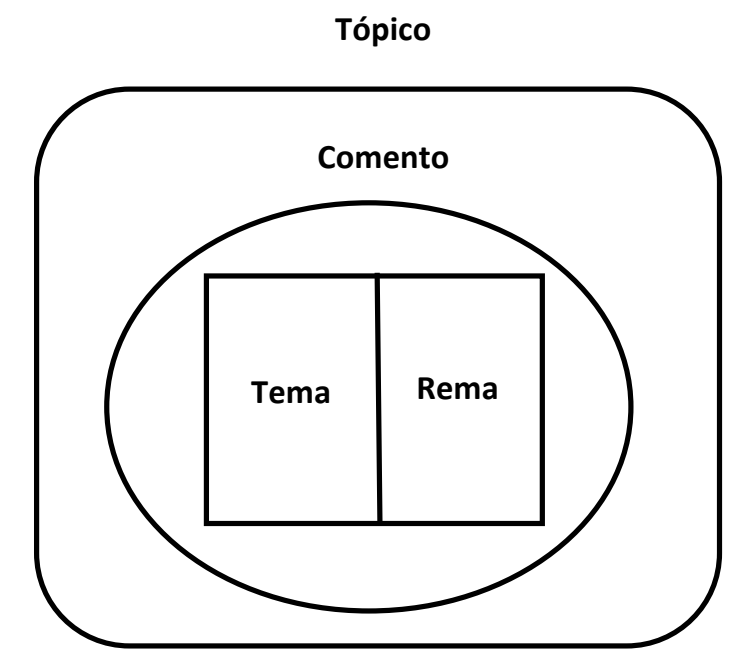

Figura 01: Relación tópico y comento

Este esquema nos permite concluir con la afirmación precisa:

[...] la oración se estructura en dos elementos esenciales que son el tópico y el comentario. El primero, es un sintagma que nos especifica sobre qué estamos hablando y el segundo es una oración completa que hace referencia un suceso relativizado a lo denotado por el tópico. (Moreno Cabrera, J. C. (2002).

\section{Tabla 2:}

\begin{tabular}{lll}
\multicolumn{1}{c}{ El tópico y el comento en la oración } \\
\hline \multicolumn{1}{c}{ Tópico } & \multicolumn{2}{c}{ Enunciado } \\
\cline { 2 - 3 } & \multicolumn{2}{c}{ Tema } \\
\hline Paykunamantaqa, & suwapunis apanman karan & Rawallutaqa. \\
\hline Según ellos, & el ladrón pudo haber hurtado & al caballo. \\
\hline Kunapunin, Sikuwanipi, & tiyayqa & wasichakushan. \\
\hline Ahora mismo, en Sicuani, & mi tía & está haciendo su casa. \\
\hline Pisqapunin, & nuqak & wayqiykunaqa. \\
\hline Exactamente cinco, & son mis & hermanos. \\
\hline Ichapascha, & chayrunaqa & suwapas kanman. \\
\hline Posiblemente, & ese hombre & pueda ser ladrón. \\
\hline Arí, & quyayqa & Killabamba llaktamanta. \\
\hline Efectivamente, & mi esposa & es de Quillabamba. \\
\hline
\end{tabular}


Características de los tópicos

Se ubica al inicio de la expresión. En vista de que el tópico debe enmarcar al comento u oración, este se ubica generalmente al inicio del enunciado como marco o circunstancial. Ejemplo.

$\frac{\text { Tópico }}{\text { Chaqraykupin, }} \frac{\text { Tema }}{\text { taytay }} \frac{\text { Rema }}{\text { sarata hallmashan. }}$
$\frac{\text { Tópico }}{\text { Paymantaqa }}, \frac{\text { Tema }}{\text { Felicianuqa }} \frac{\text { Rema }}{\text { sarata hallmashan. }}$

En caso de que la oración sea interrogativa en quechua, el tópico es marcado por el sufijo -ri y, cuando viene en posición inicial queda fuera de la interrogación separando claramente una pausa. En posición interior se convierte en soporte.

- Kay t'ikatari, ¿pitaq apamusqa? (Kay t'ikata, es tópico).

- ¿Pitaq kay t'ikatari apamusqa? (Kay t'ikatari, es soporte).

a. Es separable. Se separa del resto de la oración mediante una pausa y se marca con la coma. El tema nunca se separa con pausa del rema. Gutiérrez dice: «Los tópicos se parapetan entre pausas y, normalmente, eligen la posición inicial». (1997; 47).

$\frac{\text { Tópico }}{\text { Makinwanmi, }} \frac{\text { Comento }}{\frac{\text { Tema }}{\text { mamay } \frac{\text { Rema }}{\text { challwata hap'irparin. }}}}$

b. Es optativo. Su presencia en la oración es opcional y no obligatorio. De tal manera que se le puede sacar y el retiro no afecta a la comprensión del enunciado. Veamos:

\section{Con tópico}

$\frac{\text { Tópico }}{\text { Llaqtaypiqa, }} \frac{}{\text { llapallaykun kinuwata llank'ayku. }}$
$\frac{\text { Tópico }}{\text { Puqiypiña, }}, \frac{\text { tunasllatapas apachimusayki. }}{}$

\section{Sin tópico:}

- Llapaykun kinuwata llank'ayku. (Todos trabajamos con quinua)

- Tunasllatapas apachimusayki. (Te enviaré, aunque sea tuna)

c. No es exclusivo. En una misma secuencia se pueden incrustar más tópicos. Ejemplo:

- $\frac{\text { Tópico }}{\text { Sapallansi }} \frac{\text { Tópico }}{\text { warmakaspa }} \frac{\text { Tópico }}{\text { waq urqupi }} \frac{\text { Soporte }}{\text { Luischaqa }} \frac{\text { Aporte }}{\text { wakanta michiq. }}$

\section{Foco o relieve}

Concepto. Foco es la relevancia, prioridad, supremacía o importancia que se da a un elemento de una oración o discurso por encima de los demás componentes. Dicha relevancia obedece a la intención que tiene el hablante por dar importancia y exclusividad al segmento oracional.

La marca de foco, o importancia, se realiza primordialmente con el énfasis o mayor fuerza tonal con que el hablante pronuncia la palabra o frase que quiere destacar. Al ocurrir ello, la palabra o frase focalizada adquiere exclusividad y descarta —o excluye- a las demás negándole toda posibilidad de aparición. Claudio Pinuer, (2009), dice: «El foco es una función pragmática universal, generalizadamente codificada a través de la entonación.» 
En tal sentido, en la oración: Sumaqtapunim Rusaliyaqa punchukunata awasqa.

Si el hablante quiere destacar el encanto que siente por lo que teje Rosalía, dirá:

SUMAKTAPUNIM Rusaliyaqa punchukunata awasqa

El calificativo SUMAKTAPUNIM, es la palabra focalizada y con lo resaltado da a entender que Rosalía teje muy bien, muy hermoso, y no lo contrario.

Ahora, si quiere resaltar a Rosalía dirá.

Sumaktapunim RUSALIYAQA punchukunata awasqa

En este caso, el hablante destaca al sujeto y da a entender que es Rosalía y no otra persona.

Asimismo, si dice: Sumaktapunim Rusaliyaqa PUNCHUKUNATA awasqa.

El receptor infiere que el hablante destaca al objeto PUNCHUKUNATA y no CHALINAKUNATA, CHUMPAKUNATA ni CH'ULLUKUNATA.

Finalmente, si expresa: sumaktapunim Rusaliyaqa punchukunata AWASQA.

Notamos que el hablante destaca la acción AWASQA, y excluye otras acciones como: RANTISQA, MAÑAKUSQA, CHINKACHISQA.

\section{Procedimientos de relieve focal, focalización o énfasis}

A. Orden. La anteposición del elemento focalizado a la cabecera de la oración, al que usualmente no le corresponde esta distribución, es uno de los medios más socorridos para establecer el foco.

B. Mayor intensidad tonal. Llamado también 'prominencia prosódica' por Juan Carlos Moreno. Según Salvador Gutiérrez: «Es una sobrecarga energética que afecta a un segmento con el fin de subrayar su oposición a otro elemento generalmente no esperado» (Gutiérrez, 1997; 38).

INKA Pachakutiqsi Machupicchutaqa ruwachisqa. (El acento recae sobre la palabra inka).

$P A C H A K U ́ T I Q$ incas Machupicchutaqa ruwachisqa. (El acento recae sobre la palabra Pachakúteq).

MACHUPICCHUTAQA inka Pachakutiqsi ruwachisqa. (El acento recae sobre la palabra Machupicchutaqa).

RUWACHISQA inka Pachakutiq Machupicchutaqa. (El acento recae sobre la palabra ruwachisqa).

C. Exclusividad y exclusión. Como la focalización corresponde al plano paradigmático o in absentia, como diría Saussure — gracias a la mayor intensidad tonal con que pronuncia - el hablante logra, por una parte, focalizar o destacar el segmento in presentia y, por otra parte, excluir o descartar a las otras posibilidades latentes o in absentia, que podrían reemplazar a la unidad focalizada. En tal sentido, resaltamos un componente de la oración para, tácitamente, liquidar otra posibilidad.

INKA Pachakutiqsi Machupicchutaqa ruwachisqa. (Manan qhipukamayukchu).

PACHAKUTIQ incas Machupicchutaqa ruwachisqa. (Manan Atawallpachu).

MACHUPICCHUTAQA inka Pachakutiqsi ruwachisqa. (Manan Pikillaktatachu).

RUWACHISQA inka Pachakutiqmi Machupicchutaqa. (Manan ruwasqachu).

D. Operadores de foco. Se les denomina así a un conjunto de signos que de por sí destacan la relevancia de determinados funtivos ${ }^{1}$ de la secuencia y que a la vez generan presuposiciones, generalmente para negar su validez. Son de este tipo las preposiciones y las conjunciones. En el caso del quechua, a falta de preposiciones y conjunciones, el relieve focal se establece con los siguientes sufijos: $P A S, M I, L L A(N), \tilde{N} A, T A(N), P A Q, P I(N), W A N$, etc.

${ }^{1}$ Un funtivo es toda entidad (simple o compleja) que esté capacitada para contraer o desempeñar una función lingüística. Según la complejidad y modo de organización interna podemos diferenciar tres tipos:

- Funtivo simple: magnitud mínima capaz de contraer o desempeñar una función dada.

- Funtivo compuesto: Secuencia de dos o más funciones (cualquiera sea su organización interna) que constituye por coordinación una magnitud funcional más amplia.

- Funtivo complejo: entidad formada por la unión de dos o más entidades funcionales, bien por medio de una relación de dependencia, bien por medio de una interdependencia.

Tomado de: http://apunteslinguistica.blogspot.com/2010/11/el-lenguaje-debe-ser-un-fin-en-si-mismo.html 
- PuqyukunaPAS kawsayniyukmi kanku.

HASTA los puquiales tienen vida.

- Machullaña kaspaPAS taytayqa llank’allashanmi.

AUN siendo anciano, mi papá continúa laborando.

- WarmikumaPAS sarataqa yapuysiwankun.

INCLUSO las mujeres nos ayudan a sembrar el maíz.

- IstirLLAN liyiytaqa yachan.

Solo Esther sabe leer.

- Kay iskay irqichakunaqa ñan mihurunkuÑA.

Estos dos niños ya han comido.

- LiwiTAqatan risakqa.

A Livitaca es donde voy a ir.

- WawayPAQMI qay qulqiqa.

Para mi hijo es este dinero.

\section{Discusión de resultados}

En el quechua, como queda demostrado líneas arriba, es posible hallar unidades lingüísticas que cumplen las funciones informativas y pragmáticas de soporte/aporte, tópico/comento, y los procedimientos de focalización. Al respecto, el PhD en lingüística Cerrón-Palomino (2003; 288), asevera:

De todos los sufijos mencionados, son los validadores (fundamentalmente - $m I$, sI, -chA, -chU o sus variantes interdialectales) o el marcador de tópico - qa los que han recibido mayor atención por parte de los estudiosos. El empleo de los mismos, que en el caso de los primeros obedece a razones de relieve (señalando una información nueva), y en el segundo a la focalización de una información proporcionada de antemano, está determinado por exigencias de carácter gramatical [...] como pragmáticas [...].

Cerrón-Palomino, advierte que los sufijos quechuas de topicalización y focalización han sido estudiados. Efectivamente, uno de los primeros lingüistas cusqueños, que habla sobre este tema con conocimiento de causa es Antonio Cusihuamán (1976). Otro de los que hace referencia, aunque de forma somera, es Calvo (1993; 41), quien dice:

Esta capacidad topicalizadora del quechua contrasta con dos fenómenos generales asociados a la misma: su riqueza morfológica, que impide de hecho las expresiones ambiguas, aunque se conculque el orden general $S-O-V ;$ y la existencia de dos morfemas periféricos de carácter topicalizador (-qa) y validador focalizador (-mi/-n (ante vocal)).

En efecto, el quechua es una lengua que pocas veces se presta para la ambigüedad. Este rasgo importante es gracias a la capacidad topicalizadora. Es decir, el tópico quechua como presentador, da claridad fundamental a los enunciados.

El marcador de tópico en quechua es el sufijo - qa, cuando se trata de oraciones enunciativas, en cambio las oraciones interrogativas se topicalizan con el sufijo - ri. Este resultado coincide con lo que afirma el acertado y destacado quechuólogo cusqueño Antonio Cusihuamán, quien dice: «Hay dos enclíticos de este tipo: 1) introductivo - qa y 2) responsivo -ri. Estos enclíticos se excluyen el uno al otro en la misma oración, y su posición en la palabra es siempre final». $(1976 ; 237)$.

\section{Conclusiones}

Primera: Desde el punto de vista de la gramática funcional, el quechua es una lengua en la que es posible encontrar unidades lingüísticas con función de soporte/aporte y tópico/comento de manera muy frecuente y notoria.

Segunda: Siguiendo a Moreno Cabrera, el quechua se puede tipificar como una lengua con tópico no gramaticalizado y con sujeto conocido, al igual que el español.

Tercera: El quechua tiene como principales marcadores de tópico a los sufijos -qa y

-ri.

Cuarta: El quechua es una lengua que permite la focalización de sus unidades lingüísticas a través de la intensidad sonora.

Quinta: El quechua posee como principales operadores de foco a los sufijos -pas, $-m i,-l l a,-\tilde{n} a,-t a(n),-p a q,-p i(n),-w a n,-l l a(n),-$ $\tilde{n} a(n)$, etc.

Rev. Yachay volumen (7) Número (1), enero-diciembre 2018 


\section{Referencias bibliográficas}

Calvo Pérez, J. (1993). Pragmática y Gramática del quechua cusqueño. Cusco: Centro de Estudios Regionales Andinos Bartolomé de la Casas.

Casas Navarro, R. (2009). Introducción a la Lingüística. Lima: Edit. CEPREDIM.

Cerrón-Palomino, R. (2003). Lingüística quechua. Cusco: Editorial Centro de Estudios Regionales Andinos Bartolomé de las Casas.

Cusihuamán G., A. (1976). Gramática quechua: Cusco - Collao. Lima: Editorial Instituto de Estudios Peruanos.

Dik, Simon C. (1981). Gramática funcional. Madrid: Sociedad General Española.

Gutiérrez Ordóñez, S. (1997). Temas, remas, focos, tópicos y comentarios. Madrid: Editorial ARCO LIBROS.

Lázaro Carreter, F. (1971). Diccionario de términos filológicos. Madrid: Editorial Gredos S. A.

Lewandowski, T. (1982). Diccionario de Lingüística. Madrid: Editorial Cátedra S. A.

Luna Traill, E.; Vigueras Á. A.; Báez P. G. (2005). Diccionario Básico de Lingüística. México DF: Editorial de la Universidad Nacional Autónoma de México.

Moreno Cabrera, J. C. (2002). Curso Universitario de Lingüística General. 2.a. edición. Madrid: Editorial Síntesis.

Reguera, A. (2008). Metodología de la investigación lingüística. Buenos Aires: Editorial Brujas.

Valderrama, R y Escalante, C. (2008). Gregorio Condori Mamani/Asunta Quispe Huamán (autobiografía). Buenos Aires: Editorial Brujas. 